\title{
Cheilitis, CTCAE
}

National Cancer Institute

\section{Source}

National Cancer Institute. Cheilitis, CT CAE. NCI Thesaurus. Code C57901.

A disorder characterized by inflammation of the lip. 